\title{
New Model of 4D Einstein-Gauss-Bonnet Gravity Coupled with Nonlinear Electrodynamics
}

\author{
Sergey Il'ich Kruglov 1,2 (D) \\ 1 Department of Physics, University of Toronto, 60 St. Georges St., Toronto, ON M5S 1A7, Canada; \\ serguei.krouglov@utoronto.ca \\ 2 Department of Chemical and Physical Sciences, University of Toronto, 3359 Mississauga Road North, \\ Mississauga, ON L5L 1C6, Canada
}

\begin{abstract}
New spherically symmetric solution in 4D Einstein-Gauss-Bonnet gravity coupled with nonlinear electrodynamics is obtained. At infinity, this solution has the Reissner-Nordström behavior of the charged black hole. The black hole thermodynamics, entropy, shadow, energy emission rate, and quasinormal modes of black holes are investigated.
\end{abstract}

Keywords: Einstein-Gauss-Bonnet gravity; nonlinear electrodynamics; hawking temperature; entropy; heat capacity; black hole shadow; energy emission rate; quasinormal modes

\section{Introduction}

The heterotic string theory at the low energy limit gives the action including higher

Citation: Kruglov, S.I. New Model of 4D Einstein-Gauss-Bonnet Gravity

Coupled with Nonlinear

Electrodynamics. Universe 2021, 7, 249. https://doi.org/10.3390/ universe7070249

Academic Editors:

Panayiotis Stavrinos and

Emmanuel N. Saridakis

Received: 5 June 2021

Accepted: 12 July 2021

Published: 19 July 2021

Publisher's Note: MDPI stays neutral with regard to jurisdictional claims in published maps and institutional affiliations. order curvature terms [1-5]. Glavan and Lin proposed a new theory of gravity in four dimensions, 4D Einstein-Gauss-Bonnet gravity (4D EGB) [6], with higher-order curvature corrections. The action of the 4D EGB theory consists of the Einstein-Hilbert action and the Gauss-Bonnet (GB) term, which is a case of the Lovelock theory. The Lovelock gravity represents the generalization of Einstein's general relativity in higher dimensions that leads to covariant second-order field equations. The Einstein-Gauss-Bonnet gravity in 5D and higher dimensions was studied in [7]. Recently, 4D EGB gravity has received much attention [8-27]. Glavan and Lin showed [6] that the GB term, which is a topological invariant before regularization, while rescaling the coupling constant after regularization, contributes to the equation of motion. The authors of [12,13] found a solution of the semiclassical Einstein equations with conformal anomaly, which is also a solution in the 4D EGB gravity. The approach of Glavan and Lin was recently debated in [28-33]. It was shown by [34,35] that solutions in the 4D EGB theory are different from GR solutions as they are due to extra infinitely strongly coupled scalars. The authors of [36-38] proposed a consistent theory of 4D EGB gravity with two dynamical degrees of freedom that breaks the temporal diffeomorphism invariance, in agreement with the Lovelock theorem. In accordance with the Lovelock theorem [11], for a novel 4D theory with two degrees of freedom, the 4D diffeomorphism invariance has to be broken. In the theory of [36-38], the invariance under the 3D spatial diffeomorphism holds. The authors considered EGB gravity in arbitrary D-dimensions with the Arnowitt-Deser-Misner decomposition. Then, they regularized the Hamiltonian with counterterms, where $D-1$ diffeomorphism invariance holds and taking the limit $D \rightarrow 4$. It should be noted that the theory of [36-38], in the spherically symmetric metrics, represents the solution that is a solution in the scheme of [6] (see [39]). In this work, we obtain a black hole (BH) solution in the 4D EGB gravity coupled with nonlinear electrodynamics (NED) proposed in [40] in the framework of [36-38] theory. Quasinormal modes, deflection angle, shadows of $\mathrm{BHs}$, and Hawking radiation were studied in [41-47]. The image of the M87* BH, observed by collaboration with the Event Horizon Telescope [48], confirms the existence of $\mathrm{BHs}$ in the universe. The $\mathrm{BH}$ shadow is 
the closed curve that separates capture orbits and scattering orbits. For a review on $\mathrm{BH}$ shadows, see, for example, [49].

The paper is organized as follows. In Section 2, we find BH spherically symmetric solution in the 4D EGB gravity. It is shown that at infinity, we have the Reissner-Nordström behavior of the charged $\mathrm{BH}$. We study the $\mathrm{BH}$ thermodynamics in Section 3. The Hawking temperature and the heat capacity are calculated showing the possibility of second-order phase transitions. The entropy of BHs is obtained, which includes the area law and the logarithmic correction. In Section 4, the BH shadow is studied. The photon sphere radii, the event horizon radii, and the shadow radii are calculated. We investigate the $\mathrm{BH}$ energy emission rate in Section 5. In Section 6, quasinormal modes are studied, and we obtain the complex frequencies. In Section 7, we draw our conclusions.

\section{The Model}

The action of the EGB gravity in D-dimensions coupled with nonlinear electrodynamics (NED) is given by

$$
I=\int d^{D} x \sqrt{-g}\left[\frac{1}{16 \pi G}\left(R+\alpha \mathcal{L}_{G B}\right)+\mathcal{L}_{N E D}\right]
$$

where $\alpha$ has the dimension of (length) ${ }^{2}$, and the Lagrangian of NED, proposed in [40], is

$$
\mathcal{L}_{\text {NED }}=-\frac{\mathcal{F}}{\cosh (\sqrt[4]{|\beta \mathcal{F}|})},
$$

with the parameter $\beta(\beta \geq 0)$ having the dimension of (length) ${ }^{4}, \mathcal{F}=(1 / 4) F_{\mu v} F^{\mu v}=$ $\left(B^{2}-E^{2}\right) / 2, F_{\mu \nu}=\partial_{\mu} A_{\nu}-\partial_{\nu} A_{\mu}$ is the field strength tensor. The GB Lagrangian reads

$$
\mathcal{L}_{G B}=R^{\mu \nu \alpha \beta} R_{\mu v \alpha \beta}-4 R^{\mu v} R_{\mu v}+R^{2} .
$$

The variation of action (1) with respect to the metric results in field equations

$$
R_{\mu v}-\frac{1}{2} g_{\mu v} R+\alpha H_{\mu v}=-8 \pi G T_{\mu \nu}
$$

where

$$
H_{\mu v}=2\left(R R_{\mu v}-2 R_{\mu \alpha} R_{v}^{\alpha}-2 R_{\mu \alpha \nu \beta} R^{\alpha \beta}-R_{\mu \alpha \beta \gamma} R_{v}^{\alpha \beta \gamma}\right)-\frac{1}{2} \mathcal{L}_{G B} g_{\mu \nu} .
$$

In the following we consider a magnetic $\mathrm{BH}$ with the spherically symmetric field. The static and spherically symmetric metric in $D$ dimension is given by

$$
d s^{2}=-f(r) d t^{2}+\frac{d r^{2}}{f(r)}+r^{2} d \Omega_{D-2}^{2}
$$

where $d \Omega_{D-2}^{2}$ is the line element of the unit $(D-2)$-dimensional sphere. Equations (1) and (3)-(5) are valid in $D$ dimensions, and we will consider rescaled $\alpha$ as $\alpha \rightarrow \alpha /(D-4)$ and then the limit $D \rightarrow 4$. Taking into account that the electric charge $q_{e}=0, \mathcal{F}=q^{2} /\left(2 r^{4}\right)(q$ is a magnetic charge), one obtains the magnetic energy density [40]

$$
\rho=T_{0}^{0}=-\mathcal{L}=\frac{\mathcal{F}}{\cosh (\sqrt[4]{|\beta \mathcal{F}|})}=\frac{1}{\beta x^{4} \cosh (1 / x)},
$$

where we introduced the dimensionless variable $x=2^{1 / 4} r /\left(\beta^{1 / 4} \sqrt{q}\right)$. We consider the limit $D \rightarrow 4$ and at $\mu=v=t$ field Equation (4) gives

$$
r\left(2 \alpha f(r)-r^{2}-2 \alpha\right) f^{\prime}(r)-\left(r^{2}+\alpha f(r)-2 \alpha\right) f(r)+r^{2}-\alpha=2 r^{4} G \rho .
$$


Making use of Equation (7), we obtain

$$
\int_{0}^{r} r^{2} \rho d r=m_{M}-\frac{2^{1 / 4} q^{3 / 2}}{\beta^{1 / 4}} \arctan \left(\tanh \left(\frac{\beta^{1 / 4} \sqrt{q}}{2^{5 / 4} r}\right)\right),
$$

where the magnetic mass of the black hole reads

$$
m_{M}=\int_{0}^{\infty} r^{2} \rho d r=\frac{\pi q^{3 / 2}}{2^{7 / 4} \beta^{1 / 4}} .
$$

Then, the solution to Equation (8) is

$$
\begin{gathered}
f(r)=1+\frac{r^{2}}{2 \alpha}\left(1 \pm \sqrt{1+\frac{8 \alpha G}{r^{3}}(m+h(r))}\right), \\
h(r)=m_{M}-\frac{2^{1 / 4} q^{3 / 2}}{\beta^{1 / 4}} \arctan \left(\tanh \left(\frac{\beta^{1 / 4} \sqrt{q}}{2^{5 / 4} r}\right)\right),
\end{gathered}
$$

where $m$ is the Schwarzschild mass (the constant of integration), and $M=m+m_{M}$ is the total mass of the BH. One can verify that the Weyl tensor for the $D$-dimensional spatial part of the spherically symmetric $D$-dimensional line element (6) vanishes [39]. As a result, the new solution (11) obtained in the framework of [6] is also a solution for the consistent theory [36-38]. For Maxwell electrodynamics, the energy density is $\rho=q^{2} /\left(2 r^{4}\right)$, and Equation (8) leads to the metric function obtained in [15]. In the dimensionless form, Equation (11) becomes

$$
f(x)=1+C x^{2} \pm C \sqrt{x^{4}+x(A-B g(x))},
$$

where

$$
\begin{gathered}
A=\frac{2^{15 / 4}\left(m+m_{M}\right) \alpha G}{\beta^{3 / 4} q^{3 / 2}}, \quad B=\frac{16 \alpha G}{\beta}, \quad C=\frac{\sqrt{\beta} q}{2 \sqrt{2} \alpha}, \\
g(x)=\arctan \left(\tanh \left(\frac{1}{2 x}\right)\right),
\end{gathered}
$$

We will use the sign minus of the square root in Equations (11) and (12) (the negative branch) because, in this case, the $\mathrm{BH}$ is stable and without ghosts [8]. The asymptotic of the metric function $f(r)$ (11) for the negative branch is given by

$$
f(r)=1-\frac{2 M G}{r}+\frac{G q^{2}}{r^{2}}+\mathcal{O}\left(r^{-3}\right) \quad r \rightarrow \infty,
$$

where the total mass of the $\mathrm{BH} M=m+m_{M}$ includes the Schwarzschild mass $m$ and the electromagnetic mass $m_{M}$. According to Equation (14), the Reissner-Nordström behavior of the charged $\mathrm{BH}$ holds at infinity. It is worth noting that the limit $\beta \rightarrow 0$ has been in Equation (8) before the integration. In this case, the solution to Equation (8) at $\beta=0$ is given by [15]. The plot of the function (12) is given in Figure 1.

In accordance with Figure 1,we have two horizons-one (the extreme) horizon and no horizons-depending on the model parameters. 


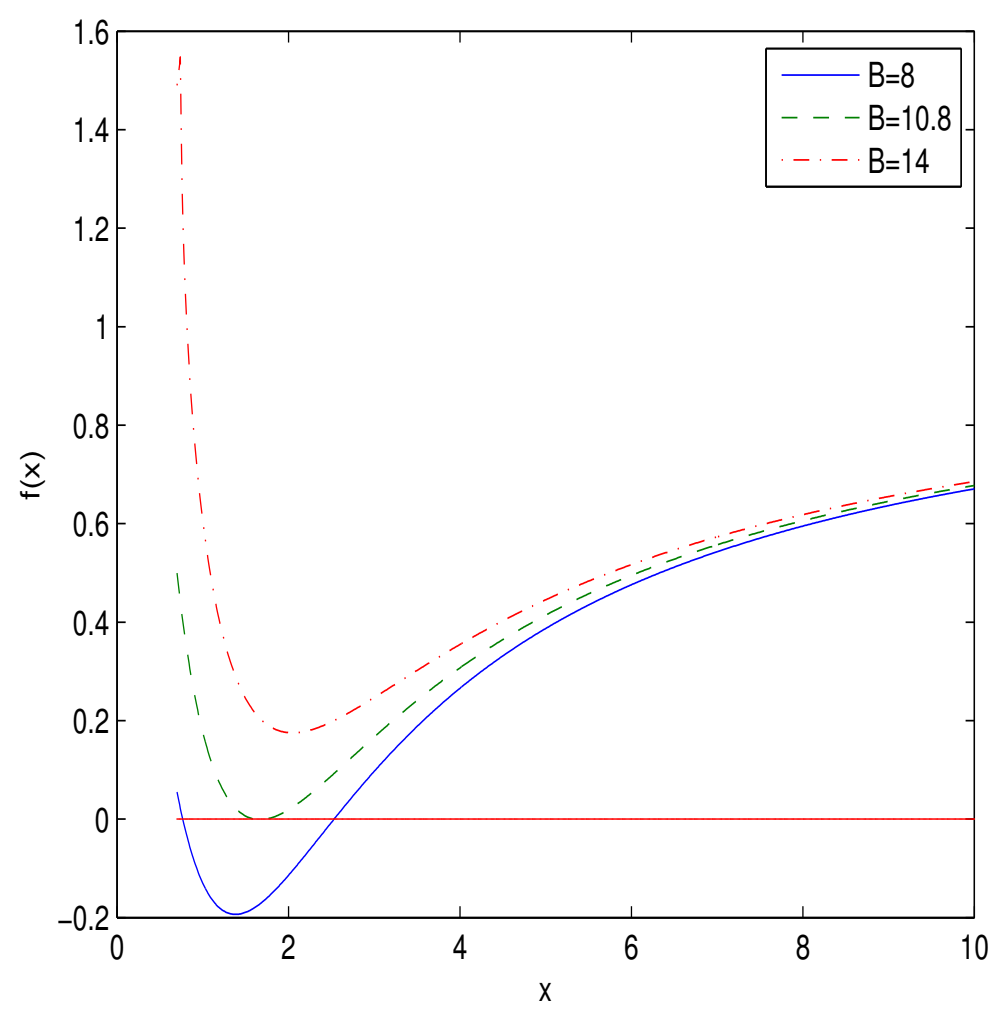

Figure 1. The plot of the function $f(x)$ for $A=7, C=1$.

\section{The BH Thermodynamics}

Consider the $\mathrm{BH}$ thermodynamics and the thermal stability of the $\mathrm{BH}$. The Hawking temperature is given by

$$
T_{H}\left(r_{+}\right)=\frac{f^{\prime}(r) \mid r=r_{+}}{4 \pi},
$$

where $r_{+}$is the event horizon radius defined by the biggest root of the equation $f\left(r_{h}\right)=0$. Making use of Equations (12) and (15), with the variable $x=2^{1 / 4} r / \sqrt[4]{\beta q^{2}}$, we obtain the Hawking temperature

$$
\begin{gathered}
T_{H}\left(x_{+}\right)=\frac{2^{1 / 4}}{4 \pi \sqrt[4]{\beta q^{2}}}\left(\frac{2 c x_{+}^{2}-1+B C^{2} x_{+}^{2} g^{\prime}\left(x_{+}\right)}{2 x_{+}\left(1+c x_{+}^{2}\right)}\right), \\
g^{\prime}\left(x_{+}\right)=-\frac{1}{2 x_{+}^{2} \cosh ^{2}\left(1 /\left(2 x_{+}\right)\right)\left(\tanh ^{2}\left(1 /\left(2 x_{+}\right)\right)+1\right)},
\end{gathered}
$$

where we substituted parameter $A$ from equation $f\left(x_{+}\right)=0$. The plot of the dimensionless function $T_{H}\left(x_{+}\right) \sqrt[4]{\beta q^{2}}$ versus $x_{+}$is depicted in Figure 2 .

According to Figure 2, the Hawking temperature is positive in some range of $x_{+}$. To study the local stability of the $\mathrm{BH}$, we calculate the heat capacity, making use of the expression

$$
C_{q}\left(x_{+}\right)=T_{H}\left(\frac{\partial S}{\partial T_{H}}\right)_{q}=\frac{\partial M\left(x_{+}\right)}{\partial T_{H}\left(x_{+}\right)}=\frac{\partial M\left(x_{+}\right) / \partial x_{+}}{\partial T_{H}\left(x_{+}\right) / \partial x_{+}},
$$

where $M\left(x_{+}\right)$is the $\mathrm{BH}$ gravitational mass depending on the event horizon radius. From equation $f\left(x_{+}\right)=0$, one obtains the BH gravitational mass

$$
M\left(x_{+}\right)=\frac{\beta^{3 / 4} q^{3 / 2}}{2^{15 / 4} \alpha G}\left(\frac{1+2 C x_{+}^{2}}{C^{2} x_{+}}+B g\left(x_{+}\right)\right) .
$$


With the aid of Equations (16) and (18), we find

$$
\begin{gathered}
\frac{\partial M\left(x_{+}\right)}{\partial x_{+}}=\frac{\beta^{3 / 4} q^{3 / 2}}{2^{15 / 4} \alpha G}\left(\frac{2 C x_{+}^{2}-1}{C^{2} x_{+}^{2}}+B g^{\prime}\left(x_{+}\right)\right), \\
\frac{\partial T_{H}\left(x_{+}\right)}{\partial x_{+}}=\frac{1}{4 \pi 2^{3 / 4} \sqrt[4]{\beta q^{2}}}\left(\frac{5 C x_{+}^{2}-2 C^{2} x_{+}^{4}+1}{x_{+}^{2}\left(1+C x_{+}^{2}\right)^{2}}\right. \\
\left.+\frac{B C^{2}\left[g^{\prime}\left(x_{+}\right)\left(1-C x_{+}^{2}\right)+x_{+} g^{\prime \prime}\left(x_{+}\right)\left(1+C x_{+}^{2}\right)\right]}{\left(1+C x_{+}^{2}\right)^{2}}\right), \\
g^{\prime \prime}\left(x_{+}\right)=\frac{\left(\tanh ^{2}\left(1 /\left(2 x_{+}\right)\right)+1\right)\left(2 x_{+}-\tanh \left(1 /\left(2 x_{+}\right)\right)\right)}{2 x_{+}^{4} \cosh ^{2}\left(1 /\left(2 x_{+}\right)\right)\left(\tanh ^{2}\left(1 /\left(2 x_{+}\right)\right)+1\right)^{2}} \\
-\frac{\tanh \left(1 /\left(2 x_{+}\right)\right)}{2 x_{+}^{4} \cosh ^{4}\left(1 /\left(2 x_{+}\right)\right)\left(\tanh ^{2}\left(1 /\left(2 x_{+}\right)\right)+1\right)^{2}} .
\end{gathered}
$$

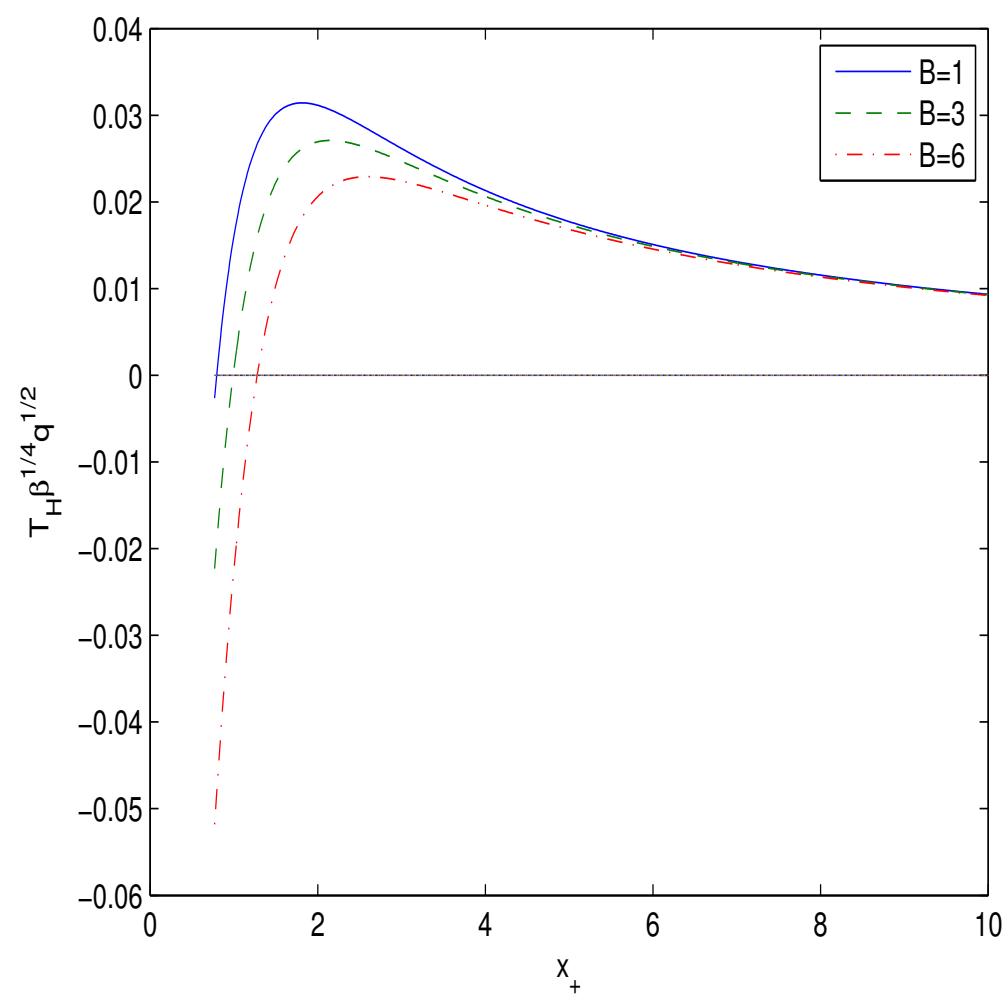

Figure 2. The plot of the function $T_{H}\left(x_{+}\right) \sqrt[4]{\beta q^{2}}$ at $C=1$.

According to Equation (17), the heat capacity possesses a singularity when the Hawking temperature has an extremum, $\partial T_{H}\left(x_{+}\right) / \partial x_{+}=0$. It follows from Equations (16) and (17) that at some point, $x_{+}=x_{1}$, the Hawking temperature and heat capacity are zero where a first-order phase transition occurs. In this point, $x_{1}$, the $\mathrm{BH}$ remnant with nonzero $\mathrm{BH}$ mass is formed, but the Hawking temperature and heat capacity become zero. In the point $x=x_{2}, \partial T_{H}\left(x_{+}\right) / \partial x_{+}=0$, the heat capacity has a discontinuity, and the second-order phase transition occurs. In the interval $x_{2}>x_{+}>x_{1}$, BHs are locally stable, and at $x_{+}>x_{2}$, the $\mathrm{BH}$ becomes unstable. By using Equations (17), (19), and (20), we represented the heat capacity in Figure 3. 


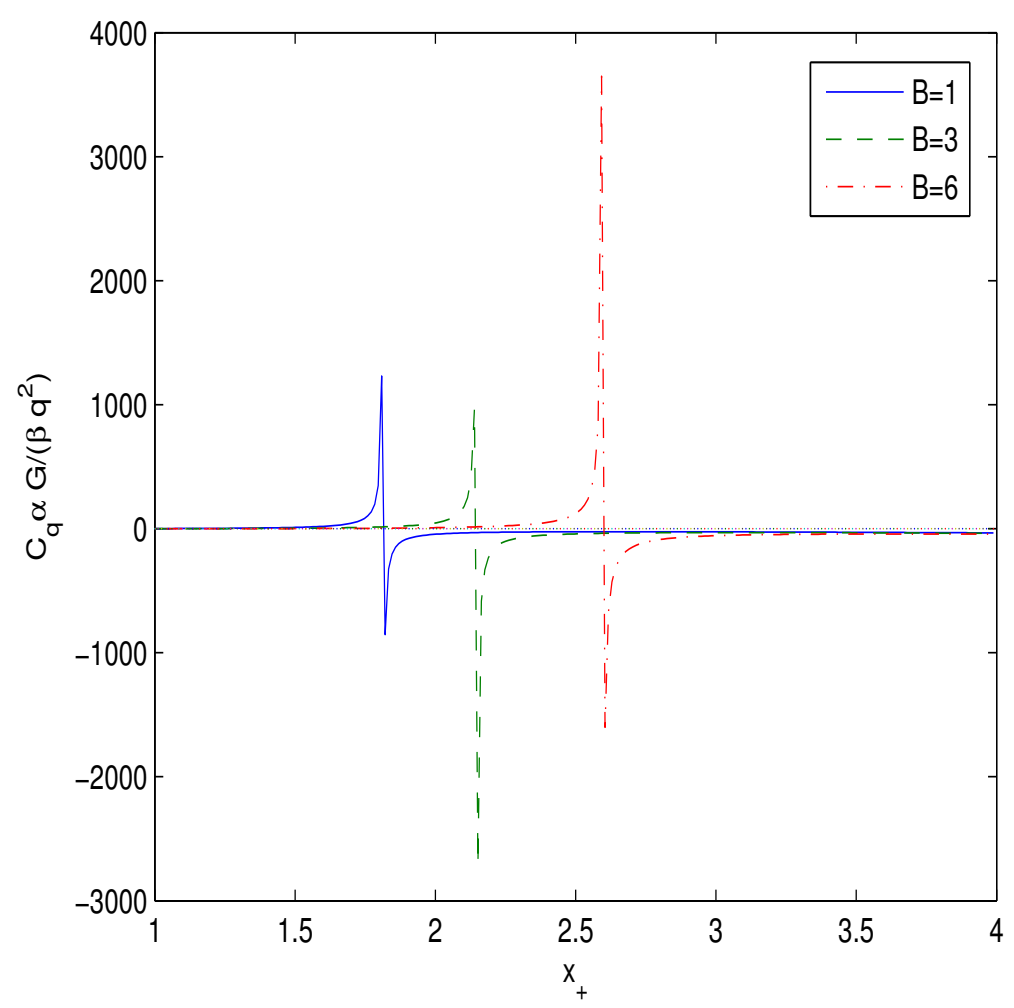

Figure 3. The plot of the function $C_{q}\left(x_{+}\right) \alpha G /\left(\beta q^{2}\right)$ at $C=1$.

In accordance with Figure 3, the BH is locally stable in the range $x_{2}>x_{+}>x_{1}$ with a positive Hawking temperature and heat capacity. The entropy $S$ at the constant charge $q$ could be calculated from the first law of BH thermodynamics $d M\left(x_{+}\right)=T_{H}\left(x_{+}\right) d S+\phi d q$,

$$
S=\int \frac{d M\left(x_{+}\right)}{T_{H}\left(x_{+}\right)}=\int \frac{1}{T_{H}\left(x_{+}\right)} \frac{\partial M\left(x_{+}\right)}{\partial x_{+}} d x_{+} .
$$

It should be noted that the entropy in this expression is defined as a constant of integration. Making use of Equations (16), (19) and (21), we obtain the entropy

$$
S=\frac{\pi \beta q^{2}}{8 C^{2} \alpha G} \int \frac{1+C x_{+}^{2}}{x_{+}} d x_{+}=\frac{\pi r_{+}^{2}}{G}+\frac{4 \pi \alpha}{G} \ln \left(\frac{\sqrt[4]{2} r_{+}}{\sqrt[4]{\beta q^{2}}}\right)+\text { Constant }
$$

where Constant is the integration constant. One can see the discussion of integration constants in [50]. We choose the integration constant as

$$
\text { Constant }=\frac{2 \pi \alpha}{G} \ln \left(\frac{\pi q \sqrt{\beta}}{\sqrt{2} G}\right) .
$$

From Equations (22) and (23), we find the BH entropy

$$
S=S_{0}+\frac{2 \pi \alpha}{G} \ln \left(S_{0}\right),
$$

where $S_{0}=\pi r_{+}^{2} / G$ is the Bekenstein-Hawking entropy. According to Equation (24), there is a logarithmic correction to area law. The entropy (24) does not contain the NED parameter $\beta$. The entropy (24) was obtained in 4D EGB gravity coupled with other NED models in [51-53]. Thus, entropy (24) does not depend on NED, which is due to the GB term in action, and the logarithmic correction vanishes when $\alpha=0$. At big $r_{+}$(event horizon radii), the Bekenstein-Hawking entropy is dominant, and for small $r_{+}$, the logarithmic correction is important. It is worth noting that at some event horizon radius $r_{0}$, the entropy 
vanishes, and when $r_{+}<r_{0}$, the entropy becomes negative. The negative entropy of BHs was discussed in [7].

\section{The Shadow of Black Holes}

The shadow of the $\mathrm{BH}$ is due to the light gravitational lensing and is a black circular disk. The image of the super-massive $\mathrm{M} 87^{*} \mathrm{BH}$ was observed by collaboration with the Event Horizon Telescope [48]. The shadow of a neutral Schwarzschild BH was investigated in [54]. The photons moving in the equatorial plane with $\vartheta=\pi / 2$ will be considered. Making use of the Hamilton-Jacobi method, the photon motion in null curves is described by the Equation (see, for example, [55])

$$
H=\frac{1}{2} g^{\mu v} p_{\mu} p_{v}=\frac{1}{2}\left(\frac{L^{2}}{r^{2}}-\frac{E^{2}}{f(r)}+\frac{\dot{r}^{2}}{f(r)}\right)=0,
$$

where $p_{\mu}$ is the photon momentum, $\dot{r}=\partial H / \partial p_{r}$, and the energy and angular momentum of a photon, which are constants of motion, are defined by $E=-p_{t}$ and $L=p_{\phi}$. Equation (25) can be represented in the form

$$
V+\dot{r}^{2}=0, \quad V=f(r)\left(\frac{L^{2}}{r^{2}}-\frac{E^{2}}{f(r)}\right) .
$$

The radius of the photon circular orbit $r_{p}$ obeys the equation $V\left(r_{p}\right)=V^{\prime}(r)_{\mid r=r_{p}}=0$. From Equation (26), one obtains

$$
\xi \equiv \frac{L}{E}=\frac{r_{p}}{\sqrt{f\left(r_{p}\right)}}, \quad f^{\prime}\left(r_{p}\right) r_{p}-2 f\left(r_{p}\right)=0,
$$

where $\xi$ is the impact parameter. The shadow radius $r_{s}$ for a distant observer, $r_{0} \rightarrow \infty$, reads $r_{s}=r_{p} / \sqrt{f\left(r_{p}\right)}$. Note that the impact parameter is $\xi=r_{s}$. The event horizon radius $r_{+}$is the biggest root of the equation $f\left(r_{h}\right)=0$. Making use of Equation (12) and $f\left(r_{h}\right)=0$, one finds the parameters $A, B$ and $C$ versus $x_{h}$

$$
\begin{gathered}
A=\frac{\left.1+2 C x_{h}^{2}+C^{2} x_{h} B g\left(x_{h}\right)\right)}{C^{2} x_{h}}, B=\frac{-1-2 C x_{h}^{2}+C^{2} x_{h} A}{\left.C^{2} x_{h} g\left(x_{h}\right)\right)}, \\
C=\frac{x_{h}^{2}+\sqrt{x_{h}^{4}+x_{h}\left(A-B g\left(x_{h}\right)\right)}}{x_{h}\left(A-B g\left(x_{h}\right)\right)},
\end{gathered}
$$

where $x_{h}=r_{h} / \sqrt[4]{\beta q^{2}}$. The plots of functions (28) are given in Figure 4.

According to Figure 4 (Subplot 1 ), if parameter $A$ increases, the event horizon radius $x_{+}$also increases. Figure 4 (Subplot 2) shows that when parameter $B$ increases, the event horizon radius decreases. According to Figure 4 (Subplot 3), if $C$ increasing the event horizon radius $x_{+}$also increasing.

In Table 1 , we presents the photon sphere radii $\left(x_{p}\right)$, the event horizon radii $\left(x_{+}\right)$, and the shadow radii $\left(x_{s}\right)$ for $A=7$ and $C=1$. The null geodesics radii $x_{p}$ belong to unstable orbits and correspond to the maximum of the potential $V(r)\left(V^{\prime \prime} \leq 0\right)$.

According to Table 1 , when the parameter $B$ increasing the shadow radius $x_{S}$ decreases. Because $x_{s}>x_{+}$, the $\mathrm{BH}$ shadow radius is given by the radius $r_{s}=x_{s} \sqrt[4]{\beta q^{2}} / 2^{1 / 4}$.

It is worth noting that nonlinear interaction of fields in the framework of NED leads to self-interaction, and photons propagate along null geodesics of the effective metric [56,57]. However, corrections in radii of photon spheres and impact parameters (due to the selfinteraction of electromagnetic fields) are small [58]. 

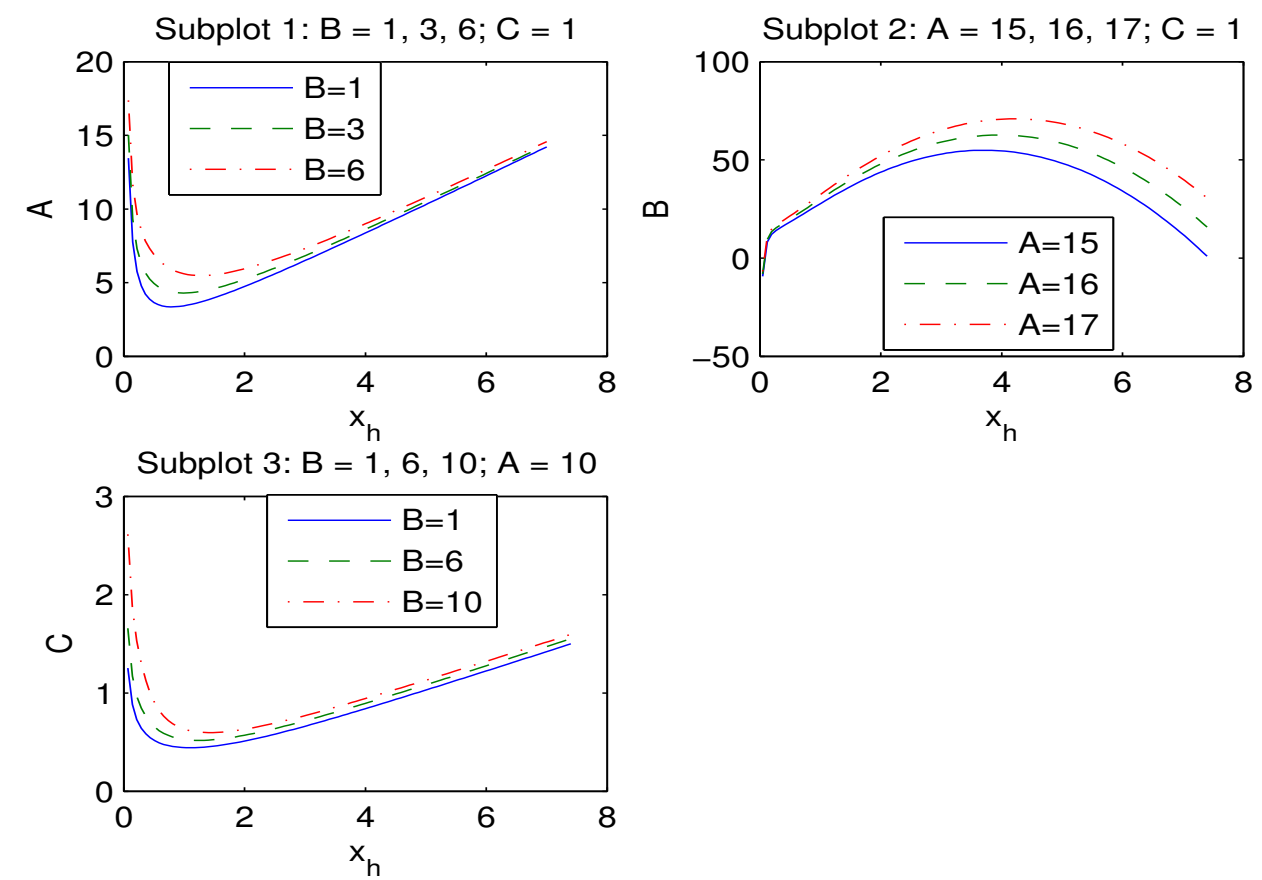

Figure 4. The plot of the functions $\mathrm{A}\left(x_{h}\right), \mathrm{B}\left(x_{h}\right), \mathrm{C}\left(x_{h}\right)$. (A) Subplot 1: $\mathrm{B}=1,3,6$; $=1$; (B) Subplot 2: $\mathrm{A}=15,16,17 ; \mathrm{C}=1 ;(\mathrm{C})$ Subplot 3: $\mathrm{B}=1,6,10 ; \mathrm{A}=10$.

Table 1. The event horizon, photon sphere and shadow dimensionless radii for $A=7, C=1$.

\begin{tabular}{ccccccccc}
\hline $\boldsymbol{B}$ & $\mathbf{0 . 1}$ & $\mathbf{0 . 5}$ & $\mathbf{1}$ & $\mathbf{2}$ & $\mathbf{3}$ & $\mathbf{4}$ & $\mathbf{5}$ & $\mathbf{6}$ \\
\hline$x_{+}$ & 3.34 & 3.31 & 3.27 & 3.19 & 3.10 & 3.01 & 2.91 & 2.80 \\
\hline$x_{p}$ & 5.11 & 5.07 & 5.02 & 4.91 & 4.80 & 4.68 & 4.55 & 4.42 \\
\hline$x_{s}$ & 8.97 & 8.92 & 8.85 & 8.71 & 8.57 & 8.43 & 8.27 & 8.11 \\
\hline
\end{tabular}

\section{The Energy Emission Rate of Black Holes}

For the observer at infinity, the $\mathrm{BH}$ shadow is linked with the high energy absorption cross section $[43,59]$. The absorption cross section, at very high energies, oscillates around the photon sphere $\sigma \approx \pi r_{s}^{2}$, and the BH energy emission rate is expressed as

$$
\frac{d^{2} E(\omega)}{d t d \omega}=\frac{2 \pi^{3} \omega^{3} r_{s}^{2}}{\exp \left(\omega / T_{H}\left(r_{+}\right)\right)-1},
$$

where $\omega$ is the emission frequency. From Equations (16) and (29), we obtain the BH energy emission rate in terms of the dimensionless variable $x_{+}=2^{1 / 4} r_{+} / \sqrt[4]{\beta q^{2}}$

$$
\beta^{1 / 4} \sqrt{q} \frac{d^{2} E(\omega)}{d t d \omega}=\frac{2 \pi^{3} \omega^{3} x_{s}^{2}}{\exp \left(\omega / \bar{T}_{H}\left(x_{+}\right)\right)-1},
$$

where $\bar{T}_{H}\left(x_{+}\right)=\beta^{1 / 4} \sqrt{q} T_{H}\left(x_{+}\right)$, and $\omega=\beta^{1 / 4} \sqrt{q} \omega$. The radiation rate, as a function of the dimensionless emission frequency $\bar{\omega}$ for $C=1, A=7$ and $B=0.1,3,6$, is plotted in Figure 5 .

According to Figure 5, we have a peak of the $\mathrm{BH}$ energy emission rate. If the parameter $B$ increases, the peak of the energy emission rate becomes smaller and is in the low frequency. At a bigger parameter $B$, the $\mathrm{BH}$ possesses a bigger lifetime. 


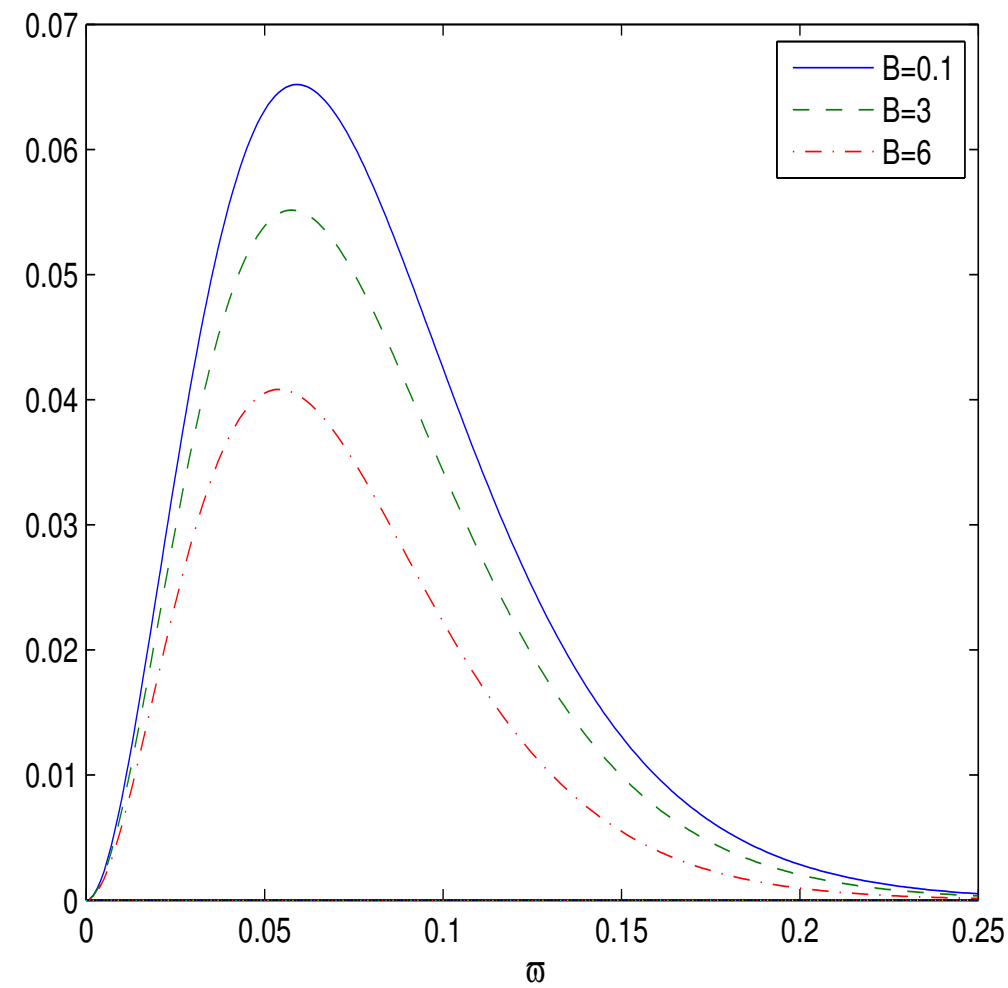

Figure 5. The plot of the function $\beta^{1 / 4} \sqrt{q} \frac{d^{2} E(\omega)}{d t d \omega}$ vs. $\omega$ for $B=0.1,3,6, A=7, C=1$.

\section{Quasinormal Modes}

Information about the stability of BHs under small perturbations can be obtained by studying quasinormal modes (QNMs), which are characterized by complex frequencies $\omega$. The mode is stable when $\operatorname{Im} \omega<0$ otherwise it is unstable. In the eikonal limit $\operatorname{Re}, \omega$ is connected with the radius of the $\mathrm{BH}$ shadow $[60,61]$. The perturbations by a scalar massless field around $\mathrm{BHs}$ are described by the effective potential barrier

$$
V(r)=f(r)\left(\frac{f^{\prime}(r)}{r}+\frac{l(l+1)}{r^{2}}\right)
$$

where $l$ is the multipole number $l=0,1,2 \ldots$ Equation (27) can be represented as

$$
V(x) \sqrt{\beta} q=\sqrt{2} f(x)\left(\frac{f^{\prime}(x)}{x}+\frac{l(l+1)}{x^{2}}\right) .
$$

The dimensionless potential $V(x) \sqrt{\beta} q$ is given in Figure 6 for $A=7, B=1, C=1$ (Subplot 1), and $l=3,4,5$ and for $A=7, C=1, l=5$, and $B=1,3,6$ (Subplot 2).

Figure 6, Subplot l, shows that the potential barriers of effective potentials have the maxima. When the $l$ increases, the height of the potential increases. According to Figure 6, Subplot 2, if the parameter $B$ increases, the height of the potential increases. The quasinormal frequencies can be found by $[60,61]$

$$
\operatorname{Re} \omega=\frac{l}{r_{s}}=\frac{l \sqrt{f\left(r_{p}\right)}}{r_{p}}, \quad \operatorname{Im} \omega=-\frac{2 n+1}{2 \sqrt{2} r_{s}} \sqrt{2 f\left(r_{p}\right)-r_{p}^{2} f^{\prime \prime}\left(r_{p}\right)},
$$

where $r_{s}$ is the $\mathrm{BH}$ shadow radius, $r_{p}$ is the $\mathrm{BH}$ photon sphere radius, and $n=0,1,2, \ldots$ is the overtone number. The frequencies, depending on parameter $B$ (at $A=7, C=1, n=1$, $l=5$ ), are represented in Table 2. 
Subplot 1: $I=3,4,5 ; A=7 ; B=1 ; C=1$

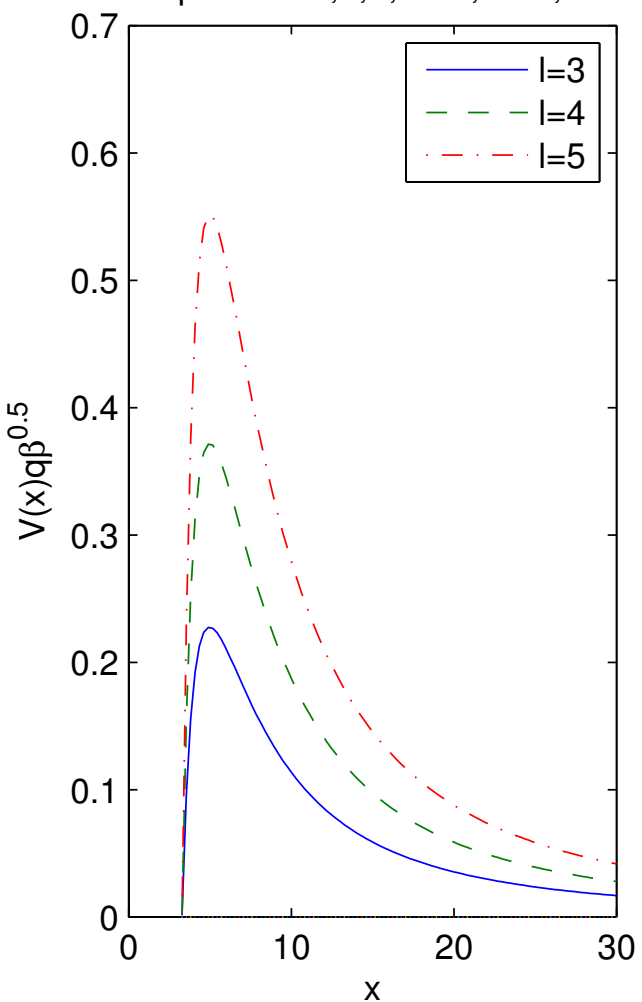

Subplot 2: $B=1,3,6 ; A=7 ; I=5 ; C=1$

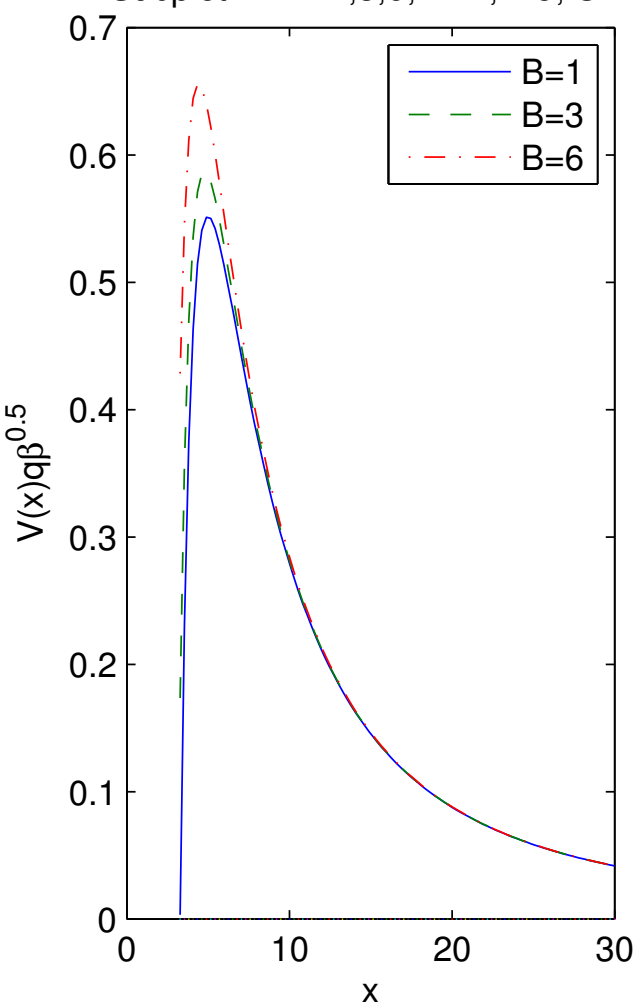

Figure 6. The plot of the function $V(x) \sqrt{\beta} q$ for $A=7, C=1$.

Table 2. The real and the imaginary parts of the frequencies vs. the parameter $B$ at $n=1, l=5$, $A=7, C=1$.

\begin{tabular}{ccccccccc}
\hline $\boldsymbol{B}$ & $\mathbf{0 . 1}$ & $\mathbf{0 . 5}$ & $\mathbf{1}$ & $\mathbf{2}$ & $\mathbf{3}$ & $\mathbf{4}$ & $\mathbf{5}$ & $\mathbf{6}$ \\
\hline$\sqrt[4]{\beta q^{2}} \operatorname{Re} \omega$ & 0.557 & 0.561 & 0.565 & 0.574 & 0.583 & 0.593 & 0.605 & 0.617 \\
\hline$-\sqrt[4]{\beta q^{2}} \operatorname{Im} \omega$ & 0.3212 & 0.3215 & 0.3221 & 0.3229 & 0.3234 & 0.3232 & 0.3230 & 0.3220 \\
\hline
\end{tabular}

The modes are stable (the real part represents the frequency of oscillations) because the imaginary parts of the frequencies in Table 2 are negative. Table 2 shows that when parameter $B$ increases the real part of the frequency $\sqrt[4]{\beta q^{2}} \operatorname{Re}, \omega$ increases, and the absolute value of the imaginary part of the frequency $\left|\sqrt[4]{\beta q^{2}} \operatorname{Im} \omega\right|$ increases. Therefore, when parameter $B$ is increased, the scalar perturbations oscillate with greater frequency and decay fast.

\section{Conclusions}

We obtained the exact spherically symmetric and magnetized BH solution in 4D EGB gravity coupled with NED. The thermodynamics and the thermal stability of magnetically charged BHs were studied by calculating the Hawking temperature and the heat capacity. The phase transitions occur in the points where the Hawking temperature possesses the extremum. It is shown that BHs are thermodynamically stable at some interval of event horizon radii when the heat capacity and the Hawking temperature are positive. The heat capacity possesses a singularity in some event horizon radii where the second-order phase transitions occur. The entropy of $\mathrm{BH}$ is calculated, including the Hawking entropy and the logarithmic correction. The photon sphere radii, the event horizon radii, and the shadow radii are calculated. We show that with increasing the model parameter $B$, the $\mathrm{BH}$ energy emission rate decreases and, as a result, the $\mathrm{BH}$ has a longer lifetime. The quasinormal modes are investigated and it is shown that increasing the parameter $B$ the 
scalar perturbations oscillate with greater frequency and decay fast. It is worth noting that other solutions in 4D EGB gravity coupled with some NED were obtained in [51-53]. It is of interest to study solutions of BHs in 4D EGB gravity coupled with different NED because astrophysical characteristics depend on them.

Funding: This research received no external funding.

Conflicts of Interest: The author declares no conflict of interest.

\section{References}

1. Gross, D.J.; Witten, E. Superstring modifications of Einstein's equations. Nucl. Phys. B 1986, 277, 1-10. [CrossRef]

2. Gross, D.J.; Sloan, J.H. The quartic effective action for the heterotic string. Nucl. Phys. B 1987, 291, 41-89. [CrossRef]

3. Metsaev, R.R.; Tseytlin, A.A. Two-loop $\beta$-function for the generalized bosonic sigma model. Phys. Lett. B 1987, 191, $354-362$. [CrossRef]

4. Zwiebach, B. Curvature squared terms and string theories. Phys. Lett. B 1985, 156, 315-317. [CrossRef]

5. Metsaev, R.R.; Tseytlin, A.A. Order $\alpha^{\prime}$ (two-loop) equivalence of the string equations of motion and the $\sigma$-model Weyl invariance conditions: Dependence on the dilaton and the antisymmetric tensor. Nucl. Phys. B 1987, 293, 385-419. [CrossRef]

6. Glavan, D.; Lin, C. Einstein-Gauss-Bonnet gravity in four-dimensional spacetime. Phys. Rev. Lett. 2020, 124, 081301. [CrossRef] [PubMed]

7. Cvetic, M.; Nojiri, S.I.; Odintsov, S.D. Black hole thermodynamics and negative entropy in de Sitter and anti-de Sitter EinsteinGauss-Bonnet gravity. Nucl. Phys. B 2002, 628, 295-330. [CrossRef]

8. Boulware, D.G.; Deser, S. String-generated gravity models. Phys. Rev. Lett. 1985, 55, 2656-2660. [CrossRef]

9. Wheeler, J.T. Symmetric solutions to the Gauss-Bonnet extended Einstein equations. Nucl. Phys. B 1986, 268, 737-746. [CrossRef]

10. Myers, R.C.; Simon, J.Z. Black-hole thermodynamics in Lovelock gravity. Phys. Rev. D 1988, 38, 2434-2444. [CrossRef]

11. Lovelock, D.J. The Einstein tensor and its generalizations. Math. Phys. 1971, 12, 498-501. [CrossRef]

12. Cai, R.G.; Cao, L.M.; Ohta, N. Black holes in gravity with conformal anomaly and logarithmic term in black hole entropy. J. High Energy Phys. 2010, 1004, 82. [CrossRef]

13. Cai, R.-G. Thermodynamics of conformal anomaly corrected black holes in AdS space. Phys. Lett. B 2014, 733, 183-189. [CrossRef]

14. Cognola, G.; Myrzakulov, R.; Sebastiani, L.; Zerbini, S. Einstein gravity with Gauss-Bonnet entropic corrections. Phys. Rev. D 2013, 88, 024006. [CrossRef]

15. Fernandes, P.G.S. Charged black holes in AdS spaces in 4D Einstein Gauss-Bonnet gravity. Phys. Lett. B 2020, 805 135468. [CrossRef]

16. Jusufi, K. Nonlinear magnetically charged black holes in 4D Einstein-Gauss—Bonnet gravity. Ann. Phys. 2020, 421, 168285. [CrossRef]

17. Ghosh, S.G.; Singh, D.V.; Kumar, R.; Maharaj, S.D. Phase transition of AdS black holes in 4D EGB gravity coupled to nonlinear electrodynamics. Ann. Phys. 2021, 424, 168347. [CrossRef]

18. Ghosh, S.G.; Maharaj, S.D. Radiating black holes in the novel 4D Einstein-Gauss-Bonnet gravity. Phys. Dark Univ. 2020, 30, 100687. [CrossRef]

19. Kumar, R.; Ghosh, S.G. Rotating black holes in 4D Einstein-Gauss-Bonnet gravity and its shadow. J. Cosmol. Astro. Phys 2020, 7, 53. [CrossRef]

20. Jin, X.H.; Gao, Y.X.; Liu, D.J. Strong gravitational lensing of a 4D Einstein-Gauss—Bonnet black hole in homogeneous plasma. Int. J. Mod. Phys. D 2020, 29, 2050065. [CrossRef]

21. Jusufi, K.; Banerjee, A.; Ghosh, S.G. Wormholes in 4D Einstein-Gauss-Bonnet gravity. Eur. Phys. J. C 2020, 80, 698. [CrossRef]

22. Guo, M.; Li, P. Innermost stable circular orbit and shadow of the 4 D Einstein-Gauss-Bonnet black hole. Eur. Phys. J. C 2020, 80, 588. [CrossRef]

23. Zhang, C.; Zhang, S.; Li, P.; Guo, M. Superradiance and stability of the regularized 4D charged Einstein-Gauss-Bonnet black hole. J. High Energy Phys. 2020, 8, 105. [CrossRef]

24. Odintsov, S.; Oikonomou, V.; Fronimos, F. Rectifying Einstein-Gauss-Bonnet inflation in view of GW170817. Nucl. Phys. B 2020, 958, 115135. [CrossRef]

25. Ai, W. A note on the novel 4D Einstein-Gauss-Bonnet gravity. Commun. Theor. Phys. 2020, 72, 095402. [CrossRef]

26. Fernandes, P.G.; Carrilho, P.; Clifton, T.; Mulryne, D.J. Derivation of regularized field equations for the Einstein-Gauss-Bonnet theory in four dimensions. Phys. Rev. D 2020, 102, 024025. [CrossRef]

27. Hennigar, R.A.; Kubiznak, D.; Mann, R.B.; Pollack, C. On taking the $D \rightarrow 4$ limit of Gauss-Bonnet gravity: Theory and solutions. J. High Energy Phys. 2020, 2020, 27. [CrossRef]

28. Gurses, M.; Sisman, T.C.; Tekin, B. Comment on "Einstein-Gauss-Bonnet Gravity in 4-Dimensional Space-Time". Phys. Rev. Lett. 2020, 125, 149001. [CrossRef]

29. Gurses, M.; Sisman, T.C.; Tekin, B. Is there a novel Einstein-Gauss-Bonnet theory in four dimensions? Eur. Phys. J. C 2020, 80, 647. [CrossRef]

30. Mahapatra, S. A note on the total action of 4D Gauss-Bonnet theory. Eur. Phys. J. C 2020, 80, 992. [CrossRef] 
31. Arrechea, J.; Delhom, A.; Jiménez-Cano, A. Inconsistencies in four-dimensional Einstein-Gauss-Bonnet gravity gravity. Chin. Phys. C 2021, 45, 013107. [CrossRef]

32. Arrechea, J.; Delhom, A.; Jiménez-Cano, A. Comment on "Einstein-Gauss-Bonnet Gravity in Four-Dimensional Spacetime". Phys. Rev. Lett. 2020, 125, 149002. [CrossRef]

33. Hohmann, M.; Pfeifer, C. Canonical variational completion and 4D Einstein-Gauss-Bonnet gravity. Eur. Phys. J. Plus 2021, 136, 180. [CrossRef]

34. Kobayashi, T. Effective scalar-tensor description of regularized Lovelock gravity in four dimensions. J. Cosmol. Astro. Phys. 2020, 7, 13. [CrossRef]

35. Bonifacio, J.; Hinterbichler, K.; Johnson, L.A. Amplitudes and 4D Gauss-Bonnet Theory. Phys. Rev. D 2020, 102, 024029. [CrossRef]

36. Aoki, K.; Gorji, M.A.; Mukohyama, S. A consistent theory of $D \rightarrow 4$ Einstein-Gauss-Bonnet gravity. Phys. Lett. B 2020, 810, 135843. [CrossRef]

37. Aoki, K.; Gorji, M.A.; Mukohyama, S. A consistent theory of $D \rightarrow 4$ Einstein-Gauss-Bonnet gravity. J. Cosmol. Astro. Phys. 2020, 2009, 14. [CrossRef]

38. Aoki, K.; Gorji, M.A.; Mizuno, S.; Mukohyama, S. Cosmology and gravitational waves in consistent $D \rightarrow 4$ Einstein-Gauss-Bonnet gravity, J. Cosmol. Astro. Phys. 2021, 2101, 54. [CrossRef]

39. Jafarzade, K.; Zangeneh, M.K.; Lobo, F.S.N. hadow, deflection angle and quasinormal modes of Born-Infeld charged black holes. J. Cosmol. Astro. Phys. 2021, 4, 8. [CrossRef]

40. Kruglov, S.I. Magnetically charged black hole in frameworkof nonlinear electrodynamics model . Int. J. Mod. Phys. A 2018, 33, 1850023. [CrossRef]

41. Konoplya, R.A.; Zinhailo, A.F. Quasinormal modes, stability and shadows of a black hole in the 4D Einstein-Gauss-Bonnet gravity. Eur. Phys. J. C 2020, 80, 1049. [CrossRef]

42. Konoplya, R.A.; Zinhailo, A.F. 4D Einstein-Lovelock black holes: Hierarchy of orders in curvature. Phys. Lett. B 2020, 807, 135607. [CrossRef]

43. Belhaj, A.; Benali, M.; Balali, A.E.; Moumni, H.E.; Ennadifi, S.E. Deflection Angle and Shadow Behaviors of Quintessential Black Holes in arbitrary Dimensions. Class. Quant. Grav. 2020, 37, 215004. [CrossRef]

44. Konoplya, R.A.; Stuchlik, Z. Are eikonal quasinormal modes linked to the unstable circular null geodesics? Phys. Lett. B 2017, 771, 597. [CrossRef]

45. Stefanov, I.Z.; Yazadjiev, S.S.; Gyulchev, G.G. Connection between black-hole quasinormal modes and lensing in the strong deflection limit. Phys. Rev. Lett. 2010, 104, 251103. [CrossRef]

46. Guo, Y.; Miao, Y.G. Null geodesics, quasinormal modes and the correspondence with shadows in high-dimensional EinsteinYang-Mills spacetimes. Phys. Rev. D 2020, 102, 084057. [CrossRef]

47. Wei, S.W.; Liu, Y.X. Null geodesics, quasinormal modes, and thermodynamic phase transition for charged black holes in asymptotically flat and dS spacetimes. Chin. Phys. C 2020, 44, 115103. [CrossRef]

48. Event Horizon Telescope Collaboration; Akiyama, K. First M87 event horizon telescope results. V. Physical origin of the asymmetric ring. Astrophys. J. 2019, 875, L5.

49. Dokuchaev, V.I.; Nazarova, N.O. Silhouettes of invisible black holes. Usp. Fiz. Nauk 2020, 190, 627. [CrossRef]

50. Medved, A.J.M.; Vagenas, E.C. When conceptual worlds collide: The GUP and the BH entropy. Phys. Rev. D 2004, 70, 124021. [CrossRef]

51. Kruglov, S.I. Einstein-Gauss-Bonnet gravity with nonlinear electrodynamics. Ann. Phys. 2021, 428, 168449. [CrossRef]

52. Kruglov, S.I. Einstein-Gauss-Bonnet Gravity with Nonlinear Electrodynamics: Entropy, Energy Emission, Quasinormal Modes and Deflection Angle. Symmetry 2021, 13, 944. [CrossRef]

53. Kruglov, S.I. Einstein-Gauss-Bonnet gravity with rational nonlinear electrodynamics. EPL 2021, 133, 69001. [CrossRef]

54. Synge, J.L. The escape of photons from gravitationally intense stars. Mon. Not. Roy. Astron. Soc. 1966, 131, 463. [CrossRef]

55. Kruglov, S.I. 4D Einstein-Gauss-Bonnet Gravity Coupled with Nonlinear Electrodynamics. Symmetry 2021, 13, 204. [CrossRef]

56. Novello, M.; Lorenci, V.A.D.; Salim, J.M.; Klippert, R. Geometrical aspects of light propagation in nonlinear electrodynamics. Phys. Rev. D 2000, 61, 045001. [CrossRef]

57. Novello, M.; Bergliaffa, S.E.P.; Salim, J.M. Singularities in general relativity coupled to nonlinear electrodynamics. Class. Quant. Grav. 2000, 17, 3821. [CrossRef]

58. Kocherlakota, P.; Rezzolla, L. Accurate mapping of spherically symmetric black holes in a parametrized framework. Phys. Rev. D 2020, 6, 064058. [CrossRef]

59. Wei, S.W.; Liu, Y.X. Observing the shadow of Einstein-Maxwell-Dilaton-Axion black hole. J. Cosmol. Astropart. Phys. 2013, 11, 63. [CrossRef]

60. Jusufi, K. Quasinormal Modes of Black Holes Surrounded by Dark Matter and Their Connection with the Shadow Radius. Phys. Rev. D 2020, 101, 084055. [CrossRef]

61. Jusufi, K. Connection Between the Shadow Radius and Quasinormal Modes in Rotating Spacetimes. Phys. Rev. D 2020, 101, 124063. [CrossRef] 